\title{
Percepção de Mães sobre a Assistência Prestada a Seus Filhos por Estudantes de Medicina da Sétima Fase: Estudo de Caso no Ambulatório de Pediatria de um Hospital Universitário
}

\author{
Mothers' Perception of the Assistance Provided \\ by Medical Students of the Seventh Semester to \\ their Children: A Case Study in a Pediatric \\ Outpatient Unit of a University Hospital
}

Gregory Vinicius Périco ${ }^{1}$ Suely Grosseman ${ }^{1}$

Ana Carolina C. Robles ${ }^{1}$ Carolina Stoll ${ }^{2}$

PALAVRAS-CHAVE:

- Relações Médico-Paciente;

- Pesquisa Qualitativa;

- Mães;

- Cuidado da Criança;

- Assistência Ambulatorial;

- Estudantes;

- Educação Médica.

KEY-WORDS:

- Physician-Patient Relationship;

- Qualitative Research;

- Mothers;

- Child Care;

- Ambulatory Care;

- Students;

- Education, Medical.

Recebido em: 07/04/2006

Aprovado em: 21/06/2006

$49 \mid \begin{aligned} & \text { REVISTA BRASILEIRA DE EDUCAÇÃO MÉDICA } \\ & 30 \text { (2) : } 49-55 ; 2006\end{aligned}$
Universidade Federal de Santa Catarina, Florianópolis, Santa Catarina, Brasil.

2 Santa Casa de Curitiba, Curitiba, Paraná, Brasil. 


\section{INTRODUÇÃO}

A satisfação do usuário tem sido incluída em estudos que avaliam a qualidade dos serviços de saúde, pois fornece informações àqueles que administram os serviços e à equipe que presta os cuidados, possibilitando desenvolver estratégias que visem à superação de limitações detectadas.

Diversos trabalhos demonstram que uma boa relação médico-paciente influencia o estado de saúde dos pacientes, já que a satisfação com o atendimento aumenta a adesão ao tratamento e, conseqüentemente, o sucesso terapêutico ${ }^{1-5}$.

Na Pediatria, a satisfação dos pais com a assistência vai influenciar a saúde de seus filhos, porque, muitas vezes, os pais são os responsáveis por levar a criança à consulta, acompanhá-la no processo de assistência e colocar em prática os aconselhamentos e tratamentos prescritos ${ }^{6}$

Em um hospital-escola, a percepção dos usuários sobre a qualidade do serviço de saúde fornece informações também sobre a qualidade do ensino. Entretanto, Zeidel e James ${ }^{7}$ afirmam que as escolas médicas que, tradicionalmente, lideram o desenvolvimento, a implementação e a disseminação de melhoramentos nos serviços, por meio dos hospitais-escola, têm se mostrado morosas na assimilação do movimento de "qualificação dos serviços". Como estes centros acadêmicos são pólos de treinamento para médicos, residentes e estudantes, seria esperado maior esforço destas entidades na realização de levantamentos acerca da percepção e expectativas do público que os utiliza.

Levando em consideração a importância de conhecer a satisfação dos usuários do serviço com o atendimento prestado por estudantes e professores, este estudo teve como objetivo conhecer a percepção de mães sobre a consulta ideal e a assistência prestada a seus filhos por estudantes de Medicina da sétima fase, no Ambulatório de Pediatria do Hospital Universitário da UFSC.

\section{MÉTODO}

Esta pesquisa utilizou o método qualitativo, do tipo estudo de caso $^{8}$.

Os sujeitos foram 12 mães cujos filhos eram consultados no Ambulatório de Pediatria do HU-UFSC. Foram incluídas mães que tivessem acompanhado seus filhos em pelo menos uma consulta anterior realizada pelos estudantes da sétima fase e que aceitassem participar do estudo. O tamanho da amostra justifica-se pela saturação dos dados a partir da décima entrevista.

O projeto do estudo foi aprovado pelo Comitê de Ética em Pesquisa com Seres Humanos da UFSC em maio de 2003. Para validação do instrumento proposto, foi realizado estudo piloto com duas mães.
Para a coleta de dados, utilizou-se entrevista semi-estruturada, em profundidade, realizada na sala de espera ou em consultórios vagos do ambulatório. Inicialmente, o teor e a finalidade da pesquisa foram explicados às mães, sendo aberta a possibilidade de indagações a respeito do estudo, bem como a opção de desistência a qualquer momento. Solicitouse que assinassem o Termo de Consentimento Livre e Esclarecido e que escolhessem um pseudônimo para garantia do anonimato.

A coleta de dados ocorreu em junho e julho de 2003. Foram coletados dados de identificação das mães e do filho, e as questões norteadoras foram: "O que você espera que um médico faça na consulta pediátrica?"; "O que você acha do atendimento realizado pelos estudantes de Medicina?"; "Por quê?"; "O que você gosta no atendimento dos estudantes?"; "Por quê?"; "O que você acha que pode ser melhorado no atendimento realizado pelos estudantes?"; "Por quê?" e "O que você sugere que possa ser feito para tanto?".

As entrevistas foram gravadas e transcritas. A análise dos dados iniciou-se a partir da primeira entrevista e foi realizada pelo processo de análise-reflexão-síntese. Neste processo, enquanto a análise decompõe os dados, a síntese os integra às diversas dimensões e contextos da vida dos sujeitos, sendo análise e síntese realizadas de forma sinérgica, por meio da reflexão, que é uma reconsideração dos dados, associando sensibilidade e razão ${ }^{9}$.

\section{RESULTADOS}

O perfil das entrevistadas está apresentado na Tabela 1.

Em relação à concepção das mães sobre a consulta ideal, vários pontos foram levantados. A atenção dispensada pelo médico foi um fator muito citado, sendo a conversa, o interesse e o fato de perguntar bastante acerca da criança considerados imprescindíveis. Nas palavras de uma das mães: "Eu espero que ele seja um bom médico, que procure saber o que meu filho tem e me explique direitinho".

Muitas ressaltaram a importância da sinceridade do médico e do seu esclarecimento na vigência de doenças, como ilustrado pelo seguinte depoimento: “Que ele não esconda nada se ele tiver algum problema!”.

A clareza do médico nas orientações fornecidas para ajudar as mães na criação de seus filhos também foi considerada fundamental na consulta.

A realização do exame físico minucioso foi citada com freqüência: "Para mim é fundamental que (...) eles façam o exame completo: nariz, boca, dente...".

O diagnóstico correto e a resolutividade da consulta foram valorizados, e a facilidade de solicitação de exames com- 
TABELA 1

Perfil das mães entrevistadas

\begin{tabular}{|c|c|c|c|c|c|c|c|}
\hline Pseudônimo & Idade & Estado Civil & $\begin{array}{l}\text { Cidade em que } \\
\text { residem }\end{array}$ & Escolaridade & $\begin{array}{l}\text { Número de } \\
\text { filhos }\end{array}$ & $\begin{array}{l}\text { Idade dos } \\
\text { filhos }\end{array}$ & $\begin{array}{c}\text { Consultas na } \\
7^{\underline{a}} \text { fase }\end{array}$ \\
\hline Francine & 21 & Casada & Florianópolis & 1ํasérie & 2 & 2 anos & 18 \\
\hline Hortênsia & 43 & Casada & Florianópolis & $5^{\mathrm{a}}$ série & 5 & 7 meses & 5 \\
\hline Carla & 28 & Solteira & Florianópolis & $2^{\underline{a}}$ série & 1 & $\begin{array}{c}1 \text { ano e } 2 \\
\text { meses }\end{array}$ & 10 \\
\hline Dora & 26 & Casada & Florianópolis & $\begin{array}{l}2^{o} \text { grau } \\
\text { completo }\end{array}$ & 2 & 6 meses & 1 \\
\hline Rosa & 45 & Casada & Florianópolis & $2^{\underline{a}}$ série & 3 & 3 meses & 1 \\
\hline Cláudia & 18 & Casada & Florianópolis & $2^{\mathrm{o}}$ ano & 1 & $\begin{array}{c}1 \text { ano e } 2 \\
\text { meses }\end{array}$ & 5 \\
\hline Adriana & 16 & Casada & Florianópolis & $7^{\mathrm{a}}$ série & 1 & 4 meses & 3 \\
\hline Maria I & 36 & Casada & Florianópolis & $8^{\underline{a}}$ série & 2 & 11 meses & 15 \\
\hline Maria II & 23 & Casada & Florianópolis & $6^{\mathrm{a}}$ série & 4 & 2 meses & 2 \\
\hline Fabiana & 28 & Casada & Florianópolis & $7^{a}$ série & 2 & 2 anos & 20 \\
\hline Marcela & 29 & Casada & $\begin{array}{c}\text { Região } \\
\text { metropolitana } \\
\text { (Florianópolis) }\end{array}$ & $\begin{array}{c}3^{o} \text { grau } \\
\text { completo }\end{array}$ & 2 & 3 meses & 3 \\
\hline Eduarda & 18 & Solteira & Florianópolis & $2^{-}$ano & 1 & 1 ano & 10 \\
\hline
\end{tabular}

plementares e de encaminhamento para atendimento mais especializado, quando necessário, mencionados por algumas

Quanto à percepção das mães sobre o atendimento no ambulatório de Pediatria do Hospital Universitário, a sua qualidade, com resolutividade e a atenção dispensada pela equipe - incluindo acolhimento do pessoal da recepção, enfermeiras, médicos e estudantes - foram motivos comumente citados para freqüentar o ambulatório. Também a relação médico-paciente-família e o comprometimento da médica foram ressaltados, como pode ser observado nos depoimentos:

Que nem tu vais na casa de uma pessoa, se tu és bem recebida tu voltas. Se tu não for, tu não quer mais saber de voltar naquele local.

Aqui, a criança é bem recebida.

Aqui, eles examinam tudo... fazem um monte de perguntas. Eu acho que assim que está certo!

Eu vou ser bem atendida e saber que eles vão vendo e que vai me resolver o problema.
Outro aspecto muito mencionado foi o acesso fácil e rápido a exames.

Outros fatores aventados pelas mães foram a presença de especialistas e a possibilidade de obter encaminhamentos com maior facilidade.

Em relação à percepção sobre a assistência prestada pelos estudantes de Medicina da sétima fase, a maioria das mães referiu estar satisfeita com o atendimento. Algumas comentaram que os estudantes atendem seus filhos "superbem".

A qualidade da atenção, não só com a criança, mas também com as mães, a paciência, a tranqüilidade e o carinho dos estudantes foram muito citados entre os aspectos do atendimento que agradam as mães: "A atenção deles, não só com ele (filho), como comigo (...) Eu acho eles bastante atenciosos e são pessoas bem calmas".

Uma entrevistada considera sua relação com os estudantes um aprendizado: "A gente aprende com eles, eles aprendem com a gente". 
A preocupação social dos acadêmicos foi assinalada: "Minha menina sempre teve problemas... Todos faziam de tudo para ajudar. Remédios para eu não pagar, eles me ajudavam".

O exame físico detalhado também foi mencionado:

Tudo que o médico-professor faz, eles (os estudantes) também fazem.

... os alunos examinam a criança "dedinho por dedinho".

Algumas valorizam o fato de vários estudantes realizarem o exame físico: "Muitas vezes, um vê uma coisa que o outro não viu: 'Olha isso aqui'. Aí eles olham de novo".

A preocupação em dar explicações claras e sanar dúvidas também foi comentada. Nas palavras de uma entrevistada: "Eu acho que aqui eles falam a língua da gente; não a língua dos médicos".

A descontração das consultas também contribui para que as mães gostem do atendimento: "Os estudantes deixam a gente à vontade, a gente conversa mais. Médico é mais sério".

Uma entrevistada aprecia o fato de os alunos reconhecerem seu filho já na sala de espera.

Quanto às limitações sentidas em relação ao atendimento, algumas referiram ter pouca confiança nos estudantes: "Junto com o médico, tudo bem! Só eles, eu não confio."

A presença de muita gente no consultório, o tempo prolongado das consultas e o número limitado de vagas foram citados, sendo sugerido que tais problemas fossem solucionados:

Eu acho que não precisa de tanta gente no consultório (...); na primeira consulta tinha sete, mais o médico, mais eu, mais meu filho (...) Isso tem que ser melhorado...

Eles fazem direitinho, só que demoram muito.

\section{DISCUSSÃO}

As expectativas das entrevistadas quanto à consulta ideal, giraram em torno, principalmente, de habilidades afetivas dos médicos. Ainda que o exame físico minucioso, o diagnóstico correto, a resolutividade da consulta, a solicitação de exames complementares e o encaminhamento para especialidades, quando necessário, tenham sido lembrados, isto não é suficiente. Elas almejam que o médico seja atencioso, converse bastante, seja sincero quanto ao diagnóstico e claro nas orientações.

Isto vem ao encontro do que afirma Jung ${ }^{10}$. Para ele, a avaliação do paciente sobre a competência do médico baseiase, predominantemente, na informação transmitida, sendo o desempenho afetivo mais valorizado que o técnico. Lewis ${ }^{11}$, revisando estudos acerca da visão dos pacientes sobre a qualidade da assistência na prática médica, verificou que a satisfação se relacionava com a competência interpessoal e técnica dos clínicos e com uma relação de parceria baseada em conversas que abordassem aspectos sociais e assuntos agradáveis.

Quanto à percepção das mães sobre o atendimento no Hospital Universitário, a maior parte dos aspectos esperados por elas numa consulta ideal foi citada, sendo muito valorizados o acolhimento e a atenção dispensada.

Na percepção sobre o atendimento pelos estudantes, a maioria das entrevistadas estava satisfeita de forma geral, e estes aspectos foram ainda mais ressaltados, sendo os mais freqüentemente mencionados: atenção dispensada, paciência, tranqüilidade e carinho demonstrado pelos estudantes; reconhecimento e abordagem das mães e crianças por eles já na sala de espera; capacidade dos estudantes de promover um clima de descontração durante a consulta; clareza nas informações prestadas; preocupação em sanar dúvidas. Estes dados demonstram a influência da relação médico-paciente em suas dimensões afetiva e comunicacional na satisfação dos usuários com a assistência.

De acordo com Auslander ${ }^{12}$ e colaboradores, a satisfação do paciente é influenciada pelo comportamento interpessoal do médico (comunicação verbal e não verbal). Entre os elementos que compõem tal comportamento estão: dar explicações claras e abrangentes e chances de o paciente falar, atitudes de empatia e acolhimento expressas pela ausência de preconceitos ou autoritarismo, demonstração de interesse pela pessoa que procura assistência e troca de informações. Para Campbell et al. ${ }^{13}$, a satisfação dos pacientes é geralmente relacionada à atitude de compreensão do médico, à sua habilidade de comunicação e ao seu desempenho técnico.

Pereira e Azevedo ${ }^{14}$ estudaram a relação médico-paciente sob a ótica de pacientes internados em Unidade de Saúde no Acre. Os entrevistados esperam que o médico seja amistoso e gentil, que saiba conversar e fornecer informações claras ao longo do tratamento e que seja solidário com o sofrimento alheio. Segundo os autores, o médico que quiser desenvolver uma relação construtiva com seu paciente precisa saber ouvilo com atenção e ter paciência e interesse, o que exige sensibilidade da parte do profissional.

Lewis ${ }^{11}$ observou que os pacientes estavam mais satisfeitos com médicos menos experientes, por considerarem que estes mostravam mais habilidades técnicas e interpessoais. Contudo, para o autor, não se pode afirmar se esta diferença de comportamento está relacionada à formação mais recente destes médicos ou a seu maior esforço, maior disponibilidade de tempo e menor desgaste profissional.

$\mathrm{Na}$ atual pesquisa, os estudantes avaliados pelas entrevistadas estão aprendendo a disciplina de Pediatria Preventiva e 
Social, sendo que a relação médico-paciente-família é valorizada e ensinada em aulas de puericultura. Além disso, não existe restrição de tempo no atendimento ambulatorial, e isto, provavelmente, influencia o esmero dos estudantes nas relações interpessoais.

Worchel et al.15, estudando a percepção de pais sobre os estilos de comunicação de pediatras, observaram que os fatores mais valorizados foram sensibilidade interpessoal, relação de parceria, informações fornecidas e individualização do tratamento por parte destes médicos. Nesse estudo, quanto mais tempo os pais passavam esclarecendo dúvidas durante as consultas, menor era o número de ligações telefônicas subseqüentes aos pediatras e menor o número de retornos.

Alguns trabalhos comentam a importância da consulta centrada no paciente e sua maior participação nas tomadas de decisão para a satisfação do usuário ${ }^{11,16-19}$.

Pesquisas demonstram que certos aspectos da relação médico-paciente - como compreensão integral do paciente, desenvolvimento da autonomia, prevenção da doença e promoção da saúde - ainda não foram bem incorporados à pratica médica ${ }^{5,20}$. Estudo de Caprara e Rodrigues ${ }^{5}$, no Programa Saúde da Família no Ceará, demonstrou que a maioria dos médicos não reconhece o paciente como sujeito co-responsável por seu estado de saúde e que, durante as consultas, os profissionais não promoviam o desenvolvimento da autonomia nem exploravam aspectos psicossociais e culturais. Os autores sugerem maior articulação entre o conhecimento técnico-científico e as representações populares do processo saúde-doença, para que se alcance maior adesão ao tratamento.

Ainda em relação à dimensão psicossocial e cultural, em estudo realizado em três municípios da Bahia, foi observado predomínio de uma escuta essencialmente biomédica da queixa do paciente e uma ação prescritiva. A investigação do problema, geralmente, foi conduzida por meio de anamnese e exame físico, sem que as condições de vida fossem exploradas e associadas à queixa principal 20 .

Pesquisas demonstram ainda que, quanto maior a diferença entre o nível de instrução do médico e das mães, maior o grau de insatisfação, devido, principalmente, à pobreza de comunicação entre eles ${ }^{12}$. Os clínicos devem ter em mente as diferentes realidades quando se fala em saúde/doença. Pacientes trazem para a inter-relação com os médicos seus próprios pontos de vista, expectativas, normas e tabus de acordo com o meio cultural em que vivem ${ }^{21}$.

Quanto aos fatores limitantes da satisfação das mães com a assistência, um dos mencionados foi o excesso de pessoas no consultório. Geralmente, grupos de quatro estudantes atendem juntos às mães e a seus filhos, sob supervisão dos médicos, o que resulta na presença de pelo menos sete indivíduos num consultório, cujas dimensões físicas não são amplas, causando congestionamento de pessoas. Portanto, compreende-se que as mães, ocasionalmente, se sintam desconfortáveis com tais circunstâncias.

A demora da consulta, que, muitas vezes, dura mais de 1 hora, ocorre devido às características do atendimento neste ambulatório. Esta é a primeira experiência dos estudantes em lidar com a puericultura, o que justifica sua menor agilidade em proceder à anamnese e ao exame físico. $\mathrm{O}$ zelo em cumprir os passos ensinados e a necessidade de supervisão e revisão dos procedimentos pelo professor prolongam ainda mais o tempo da consulta.

Para Caprara e Rodrigues ${ }^{5}$, maior tempo de consulta está associado a melhor anamnese e melhor explicação do problema e dos procedimentos diagnósticos e terapêuticos ${ }^{5}$. Por outro lado, estudos mostram que a falta de tempo e a não valorização da queixa do paciente limitam a satisfação deste com a assistência à saúde ${ }^{17}$. De acordo com Druss e Mechanic ${ }^{22,23}$, a confiança e a satisfação do usuário estão relacionadas não apenas com o tempo gasto na consulta, mas também em como este tempo preenche suas expectativas. Assim, os estudos deveriam avaliar não só a duração, mas também a qualidade do tempo gasto na consulta.

No trabalho de Pereira e Azevedo ${ }^{14}$, o tempo de duração da visita médica interferiu na satisfação com o atendimento recebido, já que, quanto maior o tempo, maior o envolvimento pessoal na consulta. Os pacientes que avaliaram a relação médico-paciente como insatisfatória estimaram o tempo de visita de seus médicos entre zero e três minutos. Já aqueles que estimaram a duração das visitas em mais de três minutos avaliaram esta relação como satisfatória.

Williams e Calnan ${ }^{24}$, em estudo sobre fatores determinantes para a satisfação dos pacientes com a prática médica na Inglaterra, verificaram que 95\% dos participantes expressavam satisfação em resposta a perguntas abertas. Porém, perguntas mais diretas revelaram aumento da insatisfação por parte dos pacientes, particularmente com respeito à relação médico-paciente: $38 \%$ sentiam que não podiam discutir problemas pessoais com seus médicos; $26 \%$ estavam insatisfeitos com a informação recebida; $25 \%$ estavam insatisfeitos com o tempo de duração das consultas e 38\% demonstraram insatisfação com o tempo de espera para as consultas.

Levando-se em consideração a complexidade da subjetividade humana no processo de ensinar-aprender e adoecercurar, observa-se a necessidade de repensar o ensino e a prática médica ${ }^{5,25}$. Neste contexto, diversas escolas têm incluído em seu currículo aspectos da comunicação como forma de 
melhorar a relação médico-paciente e, conseqüentemente, a qualidade da assistência prestada, humanizando e individualizando o atendimento ${ }^{5,25-27}$.

\section{CONSIDERAÇÕES FINAIS}

Para as mães entrevistadas, o pediatra ideal deve reunir habilidades psicomotoras, cognitivas e, principalmente, afetivas. Elas estão satisfeitas com a assistência prestada a seus filhos no Serviço Ambulatorial de Pediatria do HUUFSC, e os fatores que mais contribuíram para esta satisfação foram os interpessoais, como atenção, paciência, tranqüilidade, descontração dos estudantes e orientações claras e acessíveis.

Para obter informações, entender o contexto em que se insere a criança, aconselhar os responsáveis e tratar eventuais agravos à saúde, é preciso que o pediatra seja empático, continente com o que o responsável traz, trave uma relação de cumplicidade e se comunique efetivamente.

Se o estudante de Medicina não trouxer o atributo de ser empático e saber se comunicar de sua educação familiar, cabe à instituição de ensino sensibilizá-lo. Neste sentido, a reforma curricular do curso de Medicina da UFSC, implantada em 2003, contempla os aspectos da relação médico-paciente, fundamentais à formação do estudante de Medicina.

Quanto às limitações apontadas em relação ao atendimento, sugere-se buscar soluções que visem diminuir o número de pessoas nos consultórios e a demora das consultas.

Recomenda-se, ainda, realizar mais estudos sobre a satisfação do usuário em hospitais universitários e em outros serviços de saúde, para que possam contribuir para aprimorar sua qualidade.

\section{REFERÊNCIAS}

1. Bartlett EE, Grayson M, Barker R, Levine DM, Golden A, Libber $S$. The effects of physician communications skills on patient satisfaction; recall, and adherence. J Chronic Dis 1984; 37 (9 - 10): 755-64.

2. Linn MW, Linn BS, Stein SR. Satisfaction with ambulatory care and compliance in older patients. Med Care 1982; 20 (6): 606-14.

3. Calnan M. Towards a conceptual framework of lay evaluation of health care. Soc Sci Med 1988; 27 (9): 927-33.

4. Fitzpatrick R. Surveys of patient satisfaction: I-Important general considerations. Br Med J. 1991; 302: 887-889.

5. Caprara A, Rodrigues J. A relação assimétrica médico-paciente: repensando o vínculo terapêutico. Ciência \& Saúde Coletiva. 2004; 9 (1): 139-146.
6. Haufon N, Inkelas M, Miastry R, Olson LM. Satisfaction with health care for young children. Pediatrics 2004; 113 (6): 1965-72.

7. Zeidel ML, James BC. Improving the quality of health care in América: what medical schools, leading medical journals and federal funding agencies can do. Am J Méd. 2002; 112 (2): 165-167.

8. Bogdan RC, Biklen, SK. Investigação qualitativa em educação. Porto: Porto Editora; 1994.

9. Patrício ZM, Casagrande JM, Araújo MF (org). Qualidade de vida do trabalhador: uma abordagem qualitativa do ser humano através de novos paradigmas: Florianópolis (SC): Ed. do autor; 1999.

10. Jung HP, Horne FV, Wensing M, Hearshaw H, Grol R. Which aspects of general practioners' behavior determine patients' evaluation of care? Soc Sci Med. 1998; 47 (8): 10771087.

11. Lewis JR. Patients views on quality care in general practice: literature review. Soc Sci Med. 1994; 39 (5): 655-670.

12. Auslander WF, Thompson SJ, Dreitzer D, Santiago JV. Mothers' satisfaction with medical care: perceptions of racism, family stress, and medical outcomes in children with diabetes. Health Soc Work. 1997; 22 (3): 190-9.

13. Campbell SM, Braspenning J, Hutchinson A, Marshal M. Research methods used in developing and applying quality indicator in primary care. Qual Saf Health Care. 2002; 11: 358-64.

14. Pereira, M das G A, Azevedo, ES. A relação medico-paciente em Rio Branco/AC sob a ótica dos pacientes. Rev Assoc Méd Bras. 2005; 51 (3): 153-7.

15. Worchel FF, Prevant BC, Miner J, Alenn M, Wagner L, Nation P. Pediatricians' communication style: relationship to parents' perceptions and behaviors. J Pediatr Psychol. 1995; 20 (5): 633-44.

16. Hall JA, Roter DL, Katz NR. Meta-analysis of correlates of provider behavior in medical encounters. Med Care. 1988; 26: 657-675.

17. Cohen G. Age and health status in a patient satisfaction survey. Soc Sci Med. 1996; 42 (7): 1085-1093.

18. Simpson M, Buckman R, Stewart M, Maguire P, Lipkin M, Novack D, et al. Doctor-patient communication: the Toronto consensus statement. BMJ 1991; 303 (6814): 1385 -7.

19. Putnam SM, Stiles WB, Jacob MC, James SA. Patient exposition and physician explanation in initial medical interviews and outcomes of clinic visits. Med Care. 1985; 23 (1): 74-83. 
20. Franco ALeS, Bastos ACdeS, Alves, VS. A relação médicopaciente no Programa Saúde da Família: um estudo em três municípios do Estado da Bahia, Brasil. Cad Saúde Pública. 2005; 21 (1): 246-255.

21. Ayonrinde O. Importance of Cultural Sensitivity in Therapeutic Transactions: Considerations for Healthcare Providers [Review]. Disease Management \& Health Outcomes 2003; 11 (4): 233-248.

22. Druss B, Mechanic D. Should visit length be used as a quality indicator in primary care? The Lancet. 2003; 361: 1148.

23. Mechanic D. How should hamsters run? Some observations about sufficient patient time in primary care. BMJ 2001; 323: 266-68.

24. Williams SJ, Calnan M. Key determinants of consumer satisfactions with general practice. Fam Pract. 1991; 8 (3): 237-42.

25. Grosseman S, Patrício, ZM. A relação médico-paciente e o cuidado humano: subsídios para a promoção da educação médica. Rev Bras Educ Med. 2004. 28(2): 99-105.
26. Brody DS, Ryan K, Kuzma MA. Promoting the development of doctoring competencies in clinical settings. Fam Med. 2004;36 Suppl:S105-9.10 -

27. Egnew TR, Mauksch LB, Greer T, Farber SJ. Integrating communication training into a required family medicine clerkship. Acad Med. 2004;79(8):737-43.

\section{Conflito de Interesses}

Gregory V. Périco e Carolina Stoll fizeram a coleta de dados e a redação do manuscrito, Suely Grosseman atuou na orientação do trabalho científico e na redação do manuscrito e Ana Carolina C. Robles atuou na redação do manuscrito.

\section{Endereço para correspondência}

Gregory Vinicius Périco

Rua Lauro Linhares, 1079 - apto 201 - Trindade

88036-001 - Florianópolis - Santa Catarina

E-mail: gregoryperico@hotmail.com 